\title{
Presentation of the Fundamental Group in Digital Surfaces
}

\author{
Rémy Malgouyres \\ GREYC, ISMRA, 6, bd Mal Juin, 14000 CAEN, FRANCE. \\ E-mail: Malgouyres@greyc.ismra.fr
}

\begin{abstract}
As its analogue in the continuous framework, the digital fundamental group represents a major information on the topology of discrete objects. However, the fundamental group is an abstract information and cannot directly be encoded in a computer using its definition. A classical mathematical way to encode a discrete group is to find a presentation of this group. In this paper, we define a presentation for the fundamental group of any subset of a digital surface. This presentation can be computed by an efficient algorithm.
\end{abstract}

\section{Introduction}

As its analogue in the continuous framework, the digital fundamental group, originally introduced by T. Y. Kong in [4] in the 3D case (see also [5]), represents a major information on the topology of discrete objects. It is in particular related to the notion of a simple point in 3D ([1]), and an even closer relationship between the fundamental group and topology preservation has been established within digital surfaces $([9])$.

For these reasons, we would obtain a very powerfull tool for pattern recognition if we could make the information of the fundamental group accessible to computers in the $3 \mathrm{D}$ case. An even more difficult problem is to find an algorithm to determine whether two given 3D discrete objects have isomorphic fundamental groups. Such an algorithm would represent a significant step to determine whether two objects could be the same up to some "continuous deformation".

The fundamental group contains strictly more information than the first homology group. However, whereas the first homology group is always accessible to computers, it is somehow difficult to transform the abstract notion of the fundamental group into some data which can be handled by computers. A classical way to encode certain types of discrete groups is to find presentations of these groups. A presentation of a group is a model of the group up to isomorphism, which is characterized by an integer and some words on an alphabet called relators. Such a data can easily be encoded in a computer.

Since the problem of computing a presentation of the fundamental group seems difficult in the general case, we are lead to study it, at first, in simpler frameworks than the complete $3 \mathrm{D}$ case. Though the practical motivation comes from the $3 \mathrm{D}$ case, the study of some easier cases gives some ideas on how to 
proceed in the general case. In [7] (see also [8]), the author studies the planar $2 \mathrm{D}$ case. In this case, the fundamental group is shown to be isomorphic to a free group (i.e. there are no relators in this case). This is an ideal case since computations in the free groups are easy to perform, and the type of the group up to isomorphism is easily determined. In this paper, we study the case of digital surfaces.

Digital surfaces of three dimensional objects have proved to be a fruitful model for visualization and analysis of the objects they represent ([2]), especially in the biomedical field. Efficient algorithms for extracting surfaces from volumes, and computing shape characteristics exist $(\underline{6}])$. Sometimes, the surface itself needs to be segmented since some particular points are defined on it. Then we obtain a subset $X$ of the set of the surfels of the surface. In [9], we introduce two complementary notions of adjacency between surfels on a digital surface, which can be used to study the topology of such subsets $X$ of a digital surface, and we study the problem of topology preservation. In particular, a relationship is established between the fundamental group, and the relation between sets generated by sequential deletions of simple surfels. Here we consider the same model for the fundamental group.

We apologize for the high number of definitions necessary to the statement of the results of this paper. After having set these definitions, since the statement of the results is very technical, we first give an intuitive and non formal idea of how to proceed in the continuous case. Finally, we construct a presentation of the fundamental group of any connected subset of a digital surface. Our proof is constructive and leads to an efficient algorithm to compute this presentation.

\section{Basic Definitions and Notations}

\subsection{Groups, Normal Subgroups}

We consider a group $(\Gamma, *)$ with $1_{\Gamma}$ as unit element. Let $h \in \Gamma$. A conjugate of $h$ in $\Gamma$ is an element of $\Gamma$ of the form: $g * h * g^{-1}$ with $g \in \Gamma$. Now let $H$ be a subgroup of $\Gamma$, we say that $H$ is normal in $\Gamma$ if for any $h \in H$, any conjugate of $h$ in $\Gamma$ belongs to $H$.

Given $H$ a normal subgroup of $\Gamma$, we consider $\equiv_{H}$ the relation on $\Gamma$ defined by $\left[g \equiv_{H} g^{\prime}\right] \Longleftrightarrow\left[g^{\prime} * g^{-1} \in H\right]$. Since $H$ is a normal subgroup of $\Gamma$, this is an equivalence relation. We denote by $\Gamma / H$ the set of equivalence classes of elements of $\Gamma$ under the relation $\equiv_{H}$, and by $p_{H}: \Gamma \longrightarrow \Gamma / H$ the projection which to an element of $\Gamma$ associates its equivalence class under $\equiv_{H}$. Now, if $g \equiv_{H} g^{\prime}$ and $g_{1} \equiv_{H} g_{1}^{\prime}$, then since $H$ is normal we have $g * g_{1} \equiv_{H} g^{\prime} * g_{1}^{\prime}$. Hence the product $*$ defines an operation, which we also denote by $*$, on $\Gamma / H$. To $p_{H}(g)$ and $p_{H}\left(g^{\prime}\right)$, this operation associates $p_{H}\left(g * g^{\prime}\right)$. Obviously, the element $p_{H}\left(1_{\Gamma}\right)$ of $\Gamma / H$ is a unit element for the operation $*$ on $\Gamma / H$. Furthermore, given $g \in \Gamma$, the element $p_{H}\left(g^{-1}\right)$ of $\Gamma / H$ is an inverse for $p_{H}(g)$. Therefore, $(\Gamma / H, *)$ is a group which we call quotient group of $\Gamma$ by the normal subgroup $H$. Intuitively, all elements of $\mathrm{H}$ are collapsed with the unit element to obtain the quotient group. 
Now let $P$ be any subset of $\Gamma$. We consider the subset $H$ of $\Gamma$ composed of all products of conjugates of elements of $P$ and inverses of elements of $P$. Then $H$ is a normal subgroup of $\Gamma$ which we call normal subgroup of $\Gamma$ generated by $P$. The normal subgroup generated by $P$ is the smallest normal subgroup of $\Gamma$ which contains $P$.

\subsection{Free Group, Generators and Relations}

Now, before to introduce groups given by generators and relations, we must introduce the notion of the (non abelian) free group with $m$ generators. Let $\left\{a_{1}, \ldots, a_{m}\right\} \cup\left\{a_{1}^{-1}, \ldots, a_{m}^{-1}\right\}$ be an alphabet with $2 m$ distinct letters, and let $\mathcal{W}_{m}$ be the set of the all words over this alphabet (i.e. finite sequences of letters of the alphabet). We say that two words $w \in \mathcal{W}_{m}$ and $w^{\prime} \in \mathcal{W}_{m}$ are the same up to an elementary simplification if, either $w$ can be obtained from $w^{\prime}$ by inserting in $w^{\prime}$ a sequence of the form $a_{i} a_{i}^{-1}$ or a sequence of the form $a_{i}^{-1} a_{i}$ with $i \in$ $\{1, \ldots, m\}$, or $w^{\prime}$ can be obtained from $w$ by inserting in $w$ a sequence of the form $a_{i} a_{i}^{-1}$ or a sequence of the form $a_{i}^{-1} a_{i}$ with $i \in\{1, \ldots, m\}$. Now, two words $w \in \mathcal{W}_{m}$ and $w^{\prime} \in \mathcal{W}_{m}$ are said to be free equivalent if there is a finite sequence $w=w_{1}, \ldots, w_{k}=w^{\prime}$ of words of $\mathcal{W}_{m}$ such that for $i=2, \ldots, k$ the word $w_{i-1}$ and $w_{i}$ are the same up to an elementary simplification. This defines an equivalence relation on $\mathcal{W}_{m}$, and we denote by $\mathcal{F}_{n}$ the set of equivalence classes of this equivalence relation. If $w \in \mathcal{W}_{m}$, we denote by $\bar{w}$ the class of $w$ under the free equivalence relation. The concatenation of words defines an operation on $\mathcal{F}_{n}$ which provides $\mathcal{F}_{n}$ with a group structure. The group thus defined is called the free group with $m$ generators.

We denote by $1_{m}$ the unit element of $\mathcal{F}_{m}$, which is equal to $\bar{w}$ where $w$ is the empty word. The only result which we shall admit on the free group is the classical result that if a word $w \in L_{n}$ is such that $\bar{w}=1_{m}$ and $w$ is not the empty word, then there exists in $w$ two successive letters $a_{i} a_{i}^{-1}$ or $a_{i}^{-1} a_{i}$ with $i \in\{1, \ldots, m\}$. This remark leads to an immediate algorithm to decide whether a word $w \in L_{n}$ is such that $\bar{w}=1_{m}$. If $w=a_{i_{1}}^{\varepsilon_{1}} \cdots a_{i_{p}}^{\varepsilon_{p}}$ is a word of $\mathcal{W}_{m}$, we denote by $w^{-1}$ the word $w^{-1}=a_{i_{p}}^{-\varepsilon_{p}} \cdots a_{i_{1}}^{-\varepsilon_{1}}$. We have: $(\bar{w})^{-1}=\overline{w^{-1}}$.

Now we introduce the groups given by generators and relations. We consider $m \in \mathrm{N}^{*}$ and a finite subset $\mathcal{R}$ of $\mathcal{W}_{m}$. In this context, we call elements of $\mathcal{R}$ relators. We want to define a group from the free group with $m$ generators, in which the words of $\mathcal{R}$ represent the unit element. This group, called the group with $m$ generators and the relations of $\mathcal{R}$, is the quotient of the free group $\mathcal{F}_{m}$ by the normal subgroup generated by the set of the equivalence classes in $\mathcal{F}_{m}$ of elements of $\mathcal{R}$. Now, given $\Pi$ a group, finding a presentation of $\Pi$ is finding an (explicit) isomorphism from $\Pi$ to a group given by generators and relations.

\subsection{Connectedness in Digital Spaces}

Let $\Sigma$ be a fixed set and let $X \subset \Sigma$. We denote by card $(X)$ the number of elements of $X$ and we denote $\bar{X}=\Sigma \backslash X$. In the following, we shall define 
an adjacency relation $\alpha$ on $X$ to be an antireflexive symmetric binary relation on $X$. An $\alpha$-path with a length $p$ is a sequence $\left(x_{0}, \ldots, x_{p}\right)$ in which $x_{i-1}$ is $\alpha$-adjacent or equal to $x_{i}$ for $i=1, \ldots, p$. Such an $\alpha$-path is called closed if and only if $x_{0}=x_{n}$ and is called simple if the points $x_{i}$ for $i \in\{0 \ldots p\}$ are pairwise distinct. Given an $\alpha$-path $c=\left(x_{0}, \ldots, x_{p}\right)$, we denote by $c^{-1}$ the reversed $\alpha$-path $\left(x_{p}, \ldots, x_{0}\right)$. Two elements $x$ and $y$ are said to be $\alpha$-connected in $X$ if there exists an $\alpha$-path $\left(x_{0}, \ldots, x_{p}\right)$ in $X$ with $x_{0}=x$ and $x_{p}=y$. The $\alpha$-connectedness relation is an equivalence relation and we call $\alpha$-connected components its equivalence classes. A set is $\alpha$-adjacent to an element $x$ if at least one element of the set is $\alpha$-adjacent to $x$. We also define the $\alpha$-neighborhood $N_{\alpha}(x)$ of $x$ by $N_{\alpha}(x)=\{y \in \Sigma / y$ is $\alpha-$ adjacent to $x\}$.

\subsection{Structure of a Digital Jordan Surface}

We describe here the model of surface we consider, and some local structures which can be defined on such a surface. Afterwards, we do not consider anymore the volume from which the surface is build, and we give some intrinsic definitions and results on subsets of a surface.

First we recall some definitions, which can be found for example in [3] or [10], restricted to the three dimensional case. In the following, voxels may be seen as units cubes rather than points of $\mathrm{Z}^{3}$. We consider two kinds of adjacency between voxels. Two voxels are said to be 18 -adjacent if they share a face or an edge. They are said to be 6-adjacent if they share a face. A surfel is a pair $(c, d)$ of 6 -adjacent voxels. It can be seen as a unit square shared by $c$ and $d$. A surface is a set of surfels.

Let $O \subset \mathrm{Z}^{3}$ be a 6 -connected or 18 -connected set. We consider $\Sigma$ the set of all surfels of the form $(x, y)$ with $x \in O$ and $y \in \bar{O}$ : we call $\Sigma$ the surface of $O$. it is possible to define an adjacency relation between surfels of $\Sigma$. Such a surfel has exactly 4 neighbors, one per edge under this relation. We call this adjacency relation the $e-$ adjacency relation on the surface of $O$. Let $s$ and $s^{\prime}$ be two $e$-adjacent surfels of $\Sigma$. The surfels $s$ and $s^{\prime}$ share an edge. The pair $\left\{s, s^{\prime}\right\}$ is called an edgel. The definition of the $e$-adjacency relation depends on whether we consider $O$ as 18 -connected or 6-connected.

The kind of surface thus defined satisfies the Jordan property ([3]): a surface separates the space into two parts, one of which is 6 -connected, and the other one which is 18 -connected. This kind of surface is widely used in image analysis and manipulation.

We define a loop in $\Sigma$ as an $e$-connected component of the set of the surfels of $\Sigma$ which share a vertex $w$. For example, in Figure 1, b, we see an object with three voxels. The vertex $w$ marked with a filled circle defines two loops, one which can be seen on the figure and is composed of 3 surfels, and the other loop which is hidden and is composed of 6 surfels. Considering loops is a way to duplicate formally such vertices. We can define a unique cycle in any loop $l$ : from a pair $\left(s_{1}, s_{2}\right)$ of $e$-adjacent surfels in $l$ choose $s_{3}$ the unique surfel of $l$ which is $e$-adjacent to $s_{2}$ and which is distinct from $s_{1}$. By repeating this process we obtain a unique simple closed $e$-path of surfels which we call a parametrization 


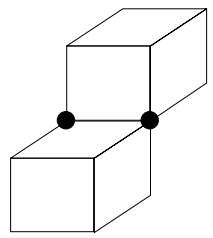

(a)

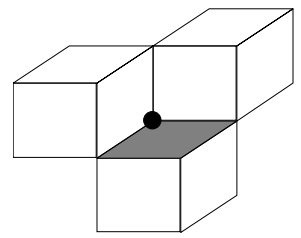

(b)

Fig. 1. Counter example and example

of the loop $l$. The length of a loop ranges between 3 and 7 . All the surfels of a loop share a common vertex. For this reason, we say that two surfels are $v$-adjacent (vertex adjacent) if they belong to a common loop.

Note that in the case of a planar surface orthogonal to one of the coordinate lines (i.e. in the case when the object $O$ is a half space), $v$-adjacency coincides with the classical $2 \mathrm{D}$ digital image notion of 8 -adjacency and $e$-adjacency to the classical 4-adjacency.

Definition 1 ( $d$-cell). We associate a dimension to surfels, edgels, and loops, which is equal respectively to 2, 1, and 0. We can identify a surfel $s$ with $\{s\}$. We call a surfel a 2-cell, an edgel a 1-cell, and a loop a 0-cell.

This dimension is compatible with the continuous analog of the digital surface. If a surfel is a member of a loop or of an edgel, we also say that it is incident to this loop or this edgel. Moreover, whenever an edgel is a subset of a loop, we also say that it is incident to this loop. We see that each surfel is incident to 4 loops and 4 edgels, and each edgel is incident to 2 loops.

In the sequel, we take the assumption that each loop of the surface of our object $O$ is a topological disk. More precisely, we assume that any two $v$-adjacent surfels which are not $e$-adjacent cannot both belong simultaneously to two given distinct loops. For instance, in Figure 1, the object (a) does not satisfy this hypothesis: The two loops corresponding to the vertices marked with filled circles contain two non $e$-adjacent surfels in common. In opposite, the object (b) satisfies our hypothesis. We can express this assumption on the object $O$ the surface of which we consider, saying that we assume that if $O$ is considered as 18-connected and $x$ and $y$ are two 18-adjacent voxels of $O$ which are not 6 -adjacent, one of the two following properties is satisfied:

1. The voxels $x$ and $y$ have an 18 -neighbor (or 6 -neighbor) in $O$ in common;

2. The voxels $x$ and $y$ have two 26-neighbors in $O$ in common which are themselves 26-adjacent.

We must have the same assumptions on $\bar{O}$ if $O$ is considered as 6 -connected.

In the sequel of this paper, we consider $\Sigma$ a fixed $e$-connected component of a digital surface, and $n \in\{e, v\}$. We also denote by $\bar{n}$ the element of $\{e, v\}$ such that $\{\bar{n}, n\}=\{e, v\}$. 


\section{Fundamental Groups, Topology Preservation}

\subsection{Simple Surfels, Homotopy}

Let $x \in \Sigma$. As we have already said, we assume that any loop in $\Sigma$ is a topological disk. However, the $v$-neighborhood of the surfel $x$ is not always a topological disk (see the $v$-neighborhood of the grey surfel of the surface of Figure 1 b for instance). If this is the case, we have to define a topology on $N_{v}(x) \cup\{x\}$ under which it is a topological disk. Let us consider two surfels $y$ and $y^{\prime}$ in $N_{v}(x) \cup\{x\}$. We say that $y$ and $y^{\prime}$ are $e_{x}$-adjacent [respectively $v_{x}$-adjacent] if they are $e$-adjacent [respectively $v$-adjacent] and are contained in a common loop which contains $x$. We denote by $G_{e}(x, X)$ [respectively $G_{v}(x, X)$ ] the graph whose vertices are the surfels of $N_{v}(x) \cap X$ and whose edges are pairs of $e_{x}$-adjacent [respectively $v_{x}$-adjacent] surfels of $N_{v}(x) \cap X$. We denote by $\mathcal{C}_{n}^{x}\left(G_{n}(x, X)\right)$ the set of all connected components of $G_{n}(x, X)$ which are $n$-adjacent to $x$. Note that $\mathcal{C}_{n}^{x}\left(G_{n}(x, X)\right)$ is a set of subsets of the set of all surfels of $\Sigma$ and not a set of surfels.

Definition 2. We call $x$ an $n$-isolated surfel if $N_{n}(x) \cap X=\emptyset$ and an $n$-interior surfel if $N_{\bar{n}}(x) \cap \bar{X}=\emptyset$.

Definition 3 (Simple surfel). A surfel $x$ is called $n$-simple in $X$ if and only if the number $\operatorname{card}\left(\mathcal{C}_{n}^{x}\left(G_{n}(x, X)\right)\right)$ of connected components of $G_{n}(x, X)$ which are $n$-adjacent to $x$ is equal to 1 , and if $x$ is not interior to $X$.

Definition 4 (homotopy). Let be $Y \subset X \subset \Sigma$. The set $Y$ is said to be (lower) $n$-homotopic to $X$ if and only if $Y$ can be obtained from $X$ by sequential deletion of $n$-simple surfels.

\subsection{The Digital Fundamental Group}

First, if $\alpha$ and $\beta$ are two $n$-paths such that the last surfel of $\alpha$ is $n$-adjacent of equal to the first surfel of $\beta$, we denote by $\alpha * \beta$ the concatenation of the two $n$-paths $\alpha$ and $\beta$.

Now we need to introduce the $n$-homotopy relation between $n$-paths. Let us consider $X \subset \Sigma$. First we introduce the notion of an elementary deformation. Two closed $n$-paths $\pi$ and $\pi^{\prime}$ in $X$ having the same extremities are said to be the same up to an elementary deformation (with fixed extremities) in $X$ if they are of the form $\pi=\pi_{1} * \gamma * \pi_{2}$ and $\pi^{\prime}=\pi_{1} * \gamma^{\prime} * \pi_{2}$, the $n$-paths $\gamma$ and $\gamma^{\prime}$ having the same extremities and being both contained in a common loop. Now, the two $n$-paths $\pi$ and $\pi^{\prime}$ are said to be $n$-homotopic (with fixed extremities) in $X$ if there exists a finite sequence of $n$-paths $\pi=\pi_{0}, \ldots, \pi_{m}=\pi^{\prime}$ such that for $i=1, \ldots, m$ the $n$-paths $\pi_{i-1}$ and $\pi_{i}$ are the same up to an elementary deformation (with fixed extremities).

Let $B \in X$ be a fixed surfel called the base surfel. We denote by $A_{B}^{n}(X)$ the set of all closed $n$-paths $\pi=\left(x_{0}, \ldots, x_{p}\right)$ which are contained in $X$ and such that $x_{0}=x_{p}=B$. The $n$-homotopy relation is an equivalence relation on 
$A_{B}^{n}(X)$, and we denote by $\Pi_{1}^{n}(X)$ the set of equivalence classes of this equivalence relation. If $c \in A_{B}^{n}(X)$ is a closed $n$-path, we denote by $[c] \in \Pi_{1}^{n}(X)$ the class of $c$ for this relation.

The concatenation of closed $n$-paths is compatible with the $n$-homotopy relation, hence it defines an operation on $\Pi_{1}^{n}(X)$, which to the class of $\alpha$ and the class of $\beta$ associates the class of $\alpha * \beta$. This operation provides $\Pi_{1}^{n}(X)$ with a group structure. We call this group the $n$-fundamental group of $X$. The $n$-fundamental group defined using a surfel $B^{\prime}$ as base surfel is isomorphic to the $n$-fundamental group defined using a surfel $B$ as base surfel if $X$ is $n$-connected.

Now we consider $Y \subset X \subset \Sigma$ and $B \in Y$ a base surfel. A closed $n$-path in $Y$ is a particular case of a closed $n$-path in $X$. Furthermore, if two closed $n$-paths of $Y$ are $n$-homotopic (with fixed extremities) in $Y$, they are $n$-homotopic (with fixed extremities) in $X$. These two properties enable us to define a canonical morphism $i_{*}: \Pi_{1}^{n}(Y) \longrightarrow \Pi_{1}^{n}(X)$, which we call the morphism induced by the inclusion map $i: Y \longrightarrow X$. To the class of a closed $n$-path $\alpha \in A_{B}^{n}(Y)$ in $\Pi_{1}^{n}(Y)$ the morphism $i_{*}$ associates the class of the same $n-$ path in $\Pi_{1}^{n}(X)$.

The following is proved in $[9]$ :

Theorem 1. Let $Y \subset X \subset \Sigma$ be $n$-connected sets. Then the two following properties are equivalent:

1. The set $Y$ is lower $n$-homotopic to $X$.

2. The morphism $i_{*}: \Pi_{1}^{n}(Y) \longrightarrow \Pi_{1}^{n}(X)$ induced by the inclusion map $i$ : $Y \longrightarrow X$ is an isomorphism and each $\bar{n}$-connected component of $\bar{Y}$ contains a surfel of $\bar{X}$.

\subsection{Homotopy in Subgraphs of $n$-adjacency Graphs}

Let $G$ be a subgraph of the $n$-adjacency graph of a set $X \in \Sigma$ with the same set of vertices. Let $\pi$ and $\pi^{\prime}$ be two paths in the graph $G$. We say that $\pi$ and $\pi^{\prime}$ are homotopic in the subgraph $G$ if there exists a finite sequence $\pi=\pi_{0}, \ldots, \pi_{m}=\pi^{\prime}$ of paths in $G$ such that for $i=1, \ldots, m$ the paths $\pi_{i-1}$ and $\pi_{i}$, as $n$-paths, are the same up to an elementary deformation (with fixed extremities). The subgraph $G$ of the $n$-adjacency graph of $X$ is called simply connected if any path in $G$ is homotopic in $G$ to a constant path of the form $(B, B)$ with $B \in X$.

\section{About the Continuous Case}

Let us first recall some definitions about $C^{1}$ surfaces embedded in $\mathrm{R}^{3}$. We denote by $D$ the opened disk of $\mathrm{R}^{2}$ with a unit radius and centered at $(0,0)$.

A continuous map $\varphi: D \longrightarrow \mathrm{R}^{3}$ is called $C^{1}$ if all the partial derivatives of $\varphi$ exist at any point $(s, t) \in \mathrm{R}^{2}$, and are continuous functions of $(s, t)$.

Let $\varphi: D \longrightarrow \mathrm{R}^{3}$ be a $C^{1}$ map.

We denote $\varphi(s, t)=\left(\varphi_{1}(s, t), \varphi_{2}(s, t), \varphi_{3}(s, t)\right) \in \mathrm{R}^{3}$. 
The map $\varphi$ is called regular if for any $(s, t) \in D$ the two vectors $\left(\frac{\partial \varphi_{1}}{\partial s}(s, t), \frac{\partial \varphi_{2}}{\partial s}(s, t), \frac{\partial \varphi_{3}}{\partial s}(s, t)\right)$ and $\left(\frac{\partial \varphi_{1}}{\partial t}(s, t), \frac{\partial \varphi_{2}}{\partial t}(s, t), \frac{\partial \varphi_{3}}{\partial t}(s, t)\right)$ are both non zero and are linearly independent in $\mathrm{R}^{3}$.

Given two metric spaces $X$ and $Y$, a continuous map $f: X \longrightarrow Y$ is called a homeomorphism if it is one to one and its inverse map is continuous.

Definition 5 ( $C^{1}$ surfaces, local parametrization). A subset $X$ of $\mathrm{R}^{3}$ is called a $C^{1}$ surface embedded in $\mathrm{R}^{3}$ (or $C^{1}$ surface for short) if for any $x \in X$ the two following properties are satisfied:

1. There exists an opened neighborhood $W_{x}$ of $x$ in $\mathrm{R}^{3}$ (we denote by $V_{x}$ the neighborhood $W_{x} \cap X$ of $x$ in $\left.X\right)$;

2. There exists a homeomorphism $\varphi_{x}: D \longrightarrow V_{x}$ which is $C^{1}$ and regular and with $\varphi_{x}((0,0))=x$.

Such a map $\varphi_{x}$ is called a local parametrization of $X$ in the neighborhood of $x$.

We have to introduce the notion of an oriented surface. Under the notions of previous Definition 5, we first consider, for $x, x^{\prime} \in X$ such that $V_{x} \cap V_{x^{\prime}} \neq \emptyset$, the map

$$
\left\{\begin{aligned}
g^{x x^{\prime}}: \varphi_{x}^{-1}\left(V_{x} \cap V_{x^{\prime}}\right) & \longrightarrow \varphi_{x^{\prime}}^{-1}\left(V_{x} \cap V_{x^{\prime}}\right) \\
(s, t) & \longmapsto \varphi_{x^{\prime}}^{-1} \circ \varphi_{x}(s, t)
\end{aligned}\right.
$$

We also denote $g^{x x^{\prime}}(s, t)=\left(g_{1}^{x x^{\prime}}(s, t), g_{2}^{x x^{\prime}}(s, t)\right) \in \mathrm{R}^{2}$. Then the two vectors $\left(\frac{\partial g_{1}^{x x^{\prime}}}{\partial s}(s, t), \frac{\partial g_{2}^{x x^{\prime}}}{\partial s}(s, t)\right)$ and $\left(\frac{\partial g_{1}^{x x^{\prime}}}{\partial t}(s, t), \frac{\partial g_{2}^{x x^{\prime}}}{\partial t}(s, t)\right)$ of $\mathrm{R}^{2}$ exist and form a basis of $\mathrm{R}^{2}$ for any $(s, t)$. An oriented surface is a couple $\left(X,\left(\varphi_{x}\right)_{x \in X}\right)$ satisfying all the above properties, and such that for any $x, x^{\prime} \in X$ such that $V_{x} \cap V_{x^{\prime}} \neq \emptyset$ and for any $(s, t) \in \varphi_{x}^{-1}\left(V_{x} \cap V_{x^{\prime}}\right)$, the basis

$$
\left(\left(\frac{\partial g_{1}^{x x^{\prime}}}{\partial s}(s, t), \frac{\partial g_{2}^{x x^{\prime}}}{\partial s}(s, t)\right),\left(\frac{\partial g_{1}^{x x^{\prime}}}{\partial t}(s, t), \frac{\partial g_{2}^{x x^{\prime}}}{\partial t}(s, t)\right)\right)
$$

of $\mathrm{R}^{2}$ is direct (i.e. has a positive orientation). Such a structure exists for any $C^{1}$ surface $X$ embedded in $\mathrm{R}^{3}$.

Let $X$ be a $C^{1}$ oriented surface. A path $c$ in $X$ is a continuous map $c$ : $[0,1] \longrightarrow X$. Such a path $c$ is called closed if its two extremities $c(0)$ and $c(1)$ are equal. A $C^{1}$ curve is a path which, as a map, admits a nowhere zero continuous derivative. A simple curve is a curve $c$ such that $\forall \alpha, \beta \in[0,1][c(\alpha)=c(\beta) \Longleftrightarrow$ $\alpha=\beta]$. A simple closed curve is a curve $c$ such that $\forall \alpha, \beta \in[0,1][c(\alpha)=$ $c(\beta) \Longleftrightarrow(\alpha=\beta$ or $\{\alpha, \beta\}=\{0,1\})$.

Let us consider two curves $c_{1}$ and $c_{2}$ in $X$ which have finitely many intersections, and such that the directions tangent to $c_{1}$ and $c_{2}$ are distinct at any of their intersections, and let $\alpha$ and $\beta$ in $[0,1]$ be such that $c_{1}(\alpha)=c_{2}(\beta)$ (in other words we consider an intersection between $c_{1}$ and $\left.c_{2}\right)$. We denote $x=c_{1}(\alpha)=c_{2}(\beta)$ the intersection point. We consider the two following vectors of $\mathrm{R}^{2}: v=\frac{d\left(\varphi_{x}^{-1} \circ c_{1}\right)}{d \alpha}(\alpha)$ and $w=\frac{d\left(\varphi_{x}^{-1} \circ c_{2}\right)}{d \beta}(\beta)$. We define the orientation number of the intersection 
$(\alpha, \beta)$ of $c_{1}$ and $c_{2}$, and we denote by $\mathcal{O}\left(\alpha, \beta, c_{1}, c_{2}\right)$, the number equal to 1 if the basis $(v, w)$ has a positive orientation in $\mathrm{R}^{2}$, and equal to -1 otherwise. This number depends on the oriented surface $\left(X,\left(\varphi_{x}\right)_{x \in X}\right)$ and not only on the $C^{1}$ surface $X$.

Now we introduce in an informal way the fundamental group of a connected subset $X$ of $\mathrm{R}^{3}$. We consider $B \in X$ a fixed point called the base point, and $A_{B}(X)$ the set of all closed paths $c$ in $X$ such that $c(0)=c(1)=B$. We introduce on $A_{B}(X)$ an equivalence relation of homotopy between paths which intuitively represents the relation of being continuously deformable one into each other inside $X$ (with fixed extremities). Then, similarly to the discrete case, we denote by $\Pi_{1}(X, B)$ the set of the equivalence classes of paths up to homotopy in $A_{B}(X)$, and the concatenation of paths defines an operation on $\Pi_{1}(X, B)$. This operation provides $\Pi_{1}(X, B)$ with a group structure, and this group is called the fundamental group of $X$. A set $X$ is called simply connected if its fundamental group reduces to a singleton (i.e. any path of $A_{B}(X)$ can be continuously deformed to a point inside $X$.

Now we can explain how to construct a presentation of the fundamental group of an oriented surface $\left(X,\left(\varphi_{x}\right)_{x \in X}\right)$. First we have to construct a finite set $\left\{\pi_{1}, \ldots, \pi_{m}\right\}$, where for any $i \in\{1, \ldots, m\}$ the element $\pi_{i}$ is either a simple curve or a simple closed curve, satisfying all the following properties :

$\left(P_{1}\right): \forall i, j \in\{1, \ldots, m\}$ with $i \neq j$ and $\left.\forall \alpha, \beta \in\right] 0,1\left[\right.$ we have $\pi_{i}(\alpha) \neq \pi_{j}(\beta)$ (in other words the curves $\pi_{i}$ can intersect only at their extremities) ;

$\left(P_{2}\right)$ : Any curve $\pi_{i}$ which is not simple closed must intersect at least another of the $\pi_{j}$ 's at each of its extremities ;

$\left(P_{3}\right)$ : The complement $X \backslash\left\{\pi_{i}(\alpha) / i=1, \ldots, m\right.$ and $\left.\alpha \in[0,1]\right\}$ of the images of all the curves $\pi_{i}$ is connected and simply connected.

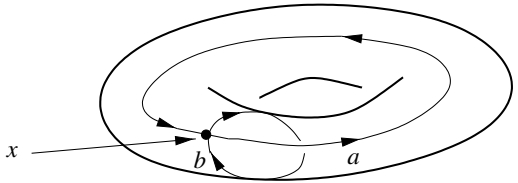

Fig. 2. Example with a torus.

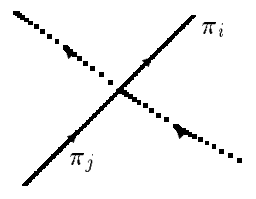

Fig. 3. An intersection point.

Let us consider for example the torus represented by Figure 2 We have represented two (simple closed) curves $\pi_{1}=a$, and $\pi_{2}=b$. All the extremities of $a$ and $b$ are at the same point $x$. The complement in the torus of these two curves is a topological disk.

Now let us consider the surface of genus 2 depicted by Figure 4 Here we have 6 curves $a, b, c, d, e$ and $f$. The curves $c$ and $e$ are simple closed and the other curves are not closed. The complements of these 6 curves in the surface 


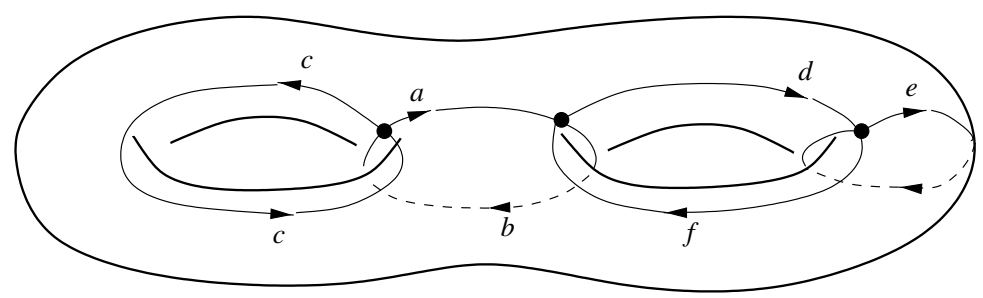

Fig. 4. Example with a surface with a genus 2 .

is a topological disk. There are three points at which we have intersections of curves.

Now let us introduce the presentation we have for the fundamental group of $X$. We consider a base point $B$ in the complement of the images of the curves $\pi_{i}$. We take $m$ generators $\left\{a_{1}, \ldots, a_{m}\right\}$, one for each of the curves $\pi_{i}$. Let us describe the words we take as relators : For any extremity $\pi_{i}(\alpha)$ with $\alpha \in\{0,1\}$ of a curve $\pi_{i}$ with $i \in\{1, \ldots, m\}$, we consider the word $R\left(\pi_{i}(\alpha)\right)$ in the $a_{j}$ 's and $a_{j}^{-1}$ 's corresponding to the curves $\pi_{j}$ which appear cyclically around the extremity $\pi_{i}(\alpha)$ (see Figure 3). The order in which the $a_{j}$ 's and $a_{j}^{-1}$ 's appear in the word $R\left(\pi_{i}(\alpha)\right)$ is precisely the cyclic order of the curves $\pi_{j}$ which meet at the point $\pi_{i}(\alpha)$. Now, whether we get in the word $R\left(\pi_{i}(\alpha)\right)$ an $a_{j}$ or an $a_{j}^{-1}$ depends on the orientation of the curve $\pi_{j}$, i.e. depends on whether $\pi_{i}(\alpha)=\pi_{j}(0)$ or $\pi_{i}(\alpha)=\pi_{j}(1)$.

For the example of the torus depicted by Figure 2 we have two generators $a$ and $b$. The $\pi_{i}$ 's all have the same extremity $x$, and the word $R(x)$ is up to conjugacy the word $a b a^{-1} b^{-1}$, commutator of $a$ and $b$.

For the example of the surface of genus 2 represented by Figure 4 we have three words $R\left(x_{1}\right), R\left(x_{2}\right)$ and $R\left(x_{3}\right)$, corresponding to the three intersection points $x_{1}, x_{2}$ and $x_{3}$ from the left to the right on the figure. We have (up to conjugacy) $R\left(x_{1}\right)=a c b^{-1} c^{-1}, R\left(x_{2}\right)=d a^{-1} f^{-1} b$, and $R\left(x_{3}\right)=e d^{-1} e^{-1} f$.

Now we consider $\mathcal{R}$ the set of all words $R\left(\pi_{i}(\alpha)\right)$ for $i=1, \ldots, m$ and $\alpha \in$ $\{0,1\}$. We denote by $\Gamma$ the group with $m$ generators and the relations of $\mathcal{R}$. The fact is that the fundamental group of the $C^{1}$ surface $X$ is isomorphic to $\Gamma$. Let us explain how the isomorphism is defined :

Let us consider a closed path $c$ of $A_{B}(X)$. First we want to construct a word $w$ in the generators. To do this, we first chose a path $c^{\prime}$, which is a curve, can be continuously deformed into $c$ inside $X$, and which has a finite intersection with all the curves $\pi_{i}, i=1, \ldots, m$ (such a curve always exists). Moreover, we may assume that at each intersection between $c^{\prime}$ and some $\pi_{i}$, the tangent directions to $c^{\prime}$ and $\pi_{i}$ are not parallel at this intersection.

Then, to construct the desired word $w\left(c^{\prime}\right)$, we go over the curve $c^{\prime}$, adding to the word we construct a symbol $a_{i}^{\mathcal{O}\left(\alpha, \beta, \pi_{i}, c^{\prime}\right)}$ each time we have an intersection $\pi_{i}(\alpha)=c^{\prime}(\beta)$. The order in which the symbols appear in the word $w\left(c^{\prime}\right)$ is the order in which the intersections with the $\pi_{i}$ 's appear along $c^{\prime}$. Then we have : 
Theorem 2. The projection of $\overline{w\left(c^{\prime}\right)} \in \mathcal{F}_{m}$ on the group $\Gamma$ depends only on the class of the path $c$ in the fundamental group. We denote this class by $\mathcal{W}(c)$, which is an element of $\Gamma$.

Theorem 3 (Presentation of the fundamental group). If we denote by $[c]$ the class of a path $c$ of $A_{B}(X)$ in the fundamental group, then the map $[c] \longmapsto \mathcal{W}(c)$ is well defined, and it is a group isomorphism from $\Pi_{1}(X, B)$ onto the group $\Gamma$.

For example, for the torus depicted by Figure 2, the fundamental group has two generators $a$ and $b$ with the single relation $a b a^{-1} b^{-1}$, and the fundamental group is therefore isomorphic to the group $\left(\mathrm{Z}^{2},+\right)$. For the example of Figure 4 , we have 6 generators and 3 relators as we noticed above.

In fact, in the continuous case, it is always possible to construct the curves $\pi_{i}$ 's in such a way that all of their extremities are at the same point. In this case, the presentation we obtain for the fundamental group has a single relation. We shall see that it does not work the same in the discrete case.

\section{The Discrete Case}

\subsection{Simple Curves on a Digital Surface}

We remind the reader that we have defined a digital surface $\Sigma$, and $n \in\{e, v\}$. In the sequel we shall assume that $n=e$ and $\bar{n}=v$. In other words, we shall analyze a fixed $e$-connected subset $X$ of the set of the surfels of $\Sigma$ with the $e$-connectivity relation, and the complement $\bar{X}$ of $X$ in $\Sigma$ with the $v$-connectivity relation. Our purpose is to compute a presentation of the $e$-fundamental group of $X$. We first want to define a set $\left\{\pi_{1}, \ldots, \pi_{m}\right\}$ of curves satisfying properties analogous to the three properties $\left(P_{1}\right),\left(P_{2}\right)$ and $\left(P_{3}\right)$ of the continuous case. The kind of curves which are to be considered here have a thickness 0 .

Definition 6 (Discrete simple curve on a surface). $A$ oriented simple curve on the surface $\Sigma$ is a sequence $\pi=\left(e_{1}, \ldots, e_{q}\right)$, with $q \geq 1$, where $e_{s}=\left(r_{s}, l_{s}\right)$ is for $s=1, \ldots, q$ a couple of surfels with $r_{s}$ e-adjacent to $l_{s}$, satisfying the three following properties :

1. For $s=1, \ldots, q-1$, the surfels $r_{s}, l_{s}, r_{s+1}$ and $l_{s+1}$ are all contained in $a$ unique common loop $L_{\pi}(s)$. For convenience, if $q=1$ we chose $L_{\pi}(0)$ and $L_{\pi}(1)$ two distinct loops which contain $r_{1}$ and $l_{1}$. If $q \geq 2$ we denote by $L_{\pi}(0)$ the unique loop which contains $r_{1}$ and $l_{1}$ and is distinct from $L_{\pi}(1)$, and we denote by $L_{\pi}(q)$ the unique loop which contains $r_{q}$ and $l_{q}$ and is distinct from $L_{\pi}(q-1)$;

2. For $s=1, \ldots, q-1$, the surfels $l_{s}$ and $l_{s+1}$ are $e$-connected in $L_{\pi}(s) \backslash\left\{r_{s}, r_{s+1}\right\}$ and the surfels $r_{s}$ and $r_{s+1}$ are $e$-connected in $L_{\pi}(s) \backslash\left\{l_{s}, l_{s+1}\right\}$;

3. The loops $L_{\pi}(s)$ for $s=0, \ldots, q$ are all distinct. 
Definition 7 (oriented simple closed curve). An oriented simple closed curve on $\Sigma$ is a sequence such as defined by the previous definition of oriented simple curves, but satisfying the following property 3' instead of the property 3 :

$3^{\prime}$ For $s, t \in\{0, \ldots, q\}$ we have $\left[L_{\pi}(s)=L_{\pi}(t) \Longleftrightarrow(s=t\right.$ or $\left.\{s, t\}=\{0, q\})\right]$.

Definition 8. Let $\pi=\left(e_{1}, \ldots, e_{q}\right)$ and $\pi^{\prime}=\left(e_{1}^{\prime}, \ldots, e_{q^{\prime}}^{\prime}\right)$ be two oriented simple curves or simple closed curves on $\Sigma$. We say that the curves $\pi$ and $\pi^{\prime}$ have no interior intersection if and only if for $s \in\{0, \ldots, q\}$ and for $t \in\left\{0, \ldots, q^{\prime}\right\}$ with $s \notin\{0, q\}$ or $s^{\prime} \notin\left\{0, q^{\prime}\right\}$ we have $: L_{\pi}(s) \neq L_{\pi^{\prime}}(t)$.

\subsection{Construction of an Appropriate set of Curves}

As we said before, we want to proceed in the discrete case as we did for the continuous case, constructing a set $\left\{\pi_{1}, \ldots, \pi_{m}\right\}$ of simple curves and simple closed curves satisfying properties analogous to the properties $\left(P_{1}\right),\left(P_{2}\right)$ and $\left(P_{3}\right)$ of the continuous case. However, in the discrete case we shall not only compute a presentation of the fundamental group of $\Sigma$, but we shall do it for any $e$-connected subset $X$ of $\Sigma$. Hence, in the sequel of this paper, $X$ denotes a set of surfels of $\Sigma$, and $B \in X$ is a fixed base surfel. We want to construct a set $\left\{\pi_{1}, \ldots, \pi_{m}\right\}$ of simple curves and simple closed curves (we denote $\pi_{i}=$ $\left(e_{1}^{i}, \ldots, e_{q_{i}}^{i}\right)$ for $i=1, \ldots, m$ and $\left.e_{s}^{i}=\left(r_{s}^{i}, l_{s}^{i}\right)\right)$, on $\Sigma$ satisfying the following properties :

$\left(P_{1}\right): \forall i, j \in\{1, \ldots, m\}$ with $i \neq j$, the curves $\pi_{i}$ and $\pi_{j}$ have no interior intersection ;

$\left(P_{2}\right)$ : For any $i \in\{1, \ldots, m\}$ and any $s \in\left\{0, q_{i}\right\}$, there exists $j \in\{1, \ldots, m\}$ and $t \in\left\{0, q_{j}\right\}$, with $i \neq j$ or $s \neq t$, such that $L_{\pi_{i}}(s)=L_{\pi_{j}}(t)$;

$\left(P_{3}\right)$ : The subgraph of the $e$-adjacency graph of $X$, obtained by removing in the $e$-adjacency graph of $X$ all the edges of the form $\left\{l_{s}^{i}, r_{s}^{i}\right\}$, is connected and simply connected.

To construct such a set of curves, we first construct a connected and simply connected subgraph of the $e$-adjacency graph of $X$, having $X$ as set of vertices. A way to do this is to consider a covering tree $G(X)$ in the $e$-adjacency graph of $X$. Such a covering tree is a connected subgraph having $X$ as set of vertices, and with no cycle so that it is simply connected. Efficient algorithms to construct covering trees in connected nonoriented graphs exist and can be found in most of books on elementary algorithms.

We can consider the set $A$ of the pairs $\left\{x_{1}, x_{2}\right\}$ of $e$-adjacent surfels of $X$, such that $\left\{x_{1}, x_{2}\right\}$ is not an edge of the subgraph $G(X)$. As we shall see, from the data of the elements of $A$, we can construct a set of oriented curves satisfying the properties $\left(P_{1}\right)$ and (since $G(X)$ is connected and simply connected) $\left(P_{3}\right)$.

Definition 9. Let $A^{\prime}$ be a set of pairs of e-adjacent surfels of $X$. An edge $\sigma \in A^{\prime}$, such that there is a loop l included in $X$ which contains $\sigma$, and such that for any $\sigma^{\prime} \in A^{\prime}$ with $\sigma^{\prime} \neq \sigma$ we have $\sigma^{\prime} \not \subset l$, is called an extremity of $A^{\prime}$. 
Because of the existence of extremities in the set $A$, the set of oriented curves we can construct from the data of $A$ does not always satisfy the property $\left(P_{2}\right)$. What we can do is remove iteratively extremities in $A$ until we get a set which contains no more extremities. More precisely, we set $A_{0}=A$, we iteratively choose an extremity $\sigma_{k}$ in the set $A_{k}$ and set $A_{k+1}=A_{k} \backslash\left\{\sigma_{k}\right\}$, until we get a set $A_{f}$ which contains no extremity. For $k=0, \ldots, f$, we denote by $G_{k}(X)$ the graph whose vertices are the surfels of $X$, an edge of $G_{k}(X)$ being either an edge of $G(X)$, or an edge of $A \backslash A_{k}$. The graph $G_{k}(X)$ is a subgraph of the $e$-adjacency graph of $X$. We have the following lemma :

Lemma 1. For $k=0, \ldots, f$, the graph $G_{k}(X)$ is connected and simply connected.

There remains to construct the oriented curves $\pi_{i}$ 's from the data of $A_{f}$. Let us consider $\sigma=\left\{r_{1}, l_{1}\right\}$ an edge of $A_{f}$. We denote $e_{1}=\left(r_{1}, l_{1}\right)$. Then the sequence $\left(e_{1}\right)$ with a single term is a simple curve on $\Sigma$. Hence we can construct a set of simple curves and simple closed curves $\left\{\pi_{1}, \ldots, \pi_{m}\right\}$ such that, denoting $\pi_{i}=\left(e_{1}^{i}, \ldots, e_{q_{i}}^{i}\right)$ for $i=1, \ldots, m$ and $e_{s}^{i}=\left(r_{s}^{i}, l_{s}^{i}\right)$, the map $\Phi:\left(r_{s}^{i}, l_{s}^{i}\right) \longmapsto$ $\left\{r_{s}^{i}, l_{s}^{i}\right\}$ is a one to one correspondence between the set $\left\{e_{s}^{i} / i=1, \ldots, m\right.$ and $\left.s=1, \ldots, q_{i}\right\}$ and the set $A_{f}$. We can for instance consider curves reduced to one edge, but it is also possible to consider longer curves, by extending the curves in loops containing exactly two edges of $A_{f}$, so that we get a lower value of $m$. By constructing the simple curves and the simple closed curves $\pi_{i}$ in this way, the $\pi_{i}$ 's satisfy the property $\left(P_{1}\right)$. Moreover, since, from its very construction, $A_{f}$ has no extremity, the $\pi_{i}$ 's satisfy the property $\left(P_{2}\right)$. At last, from Lemma 1. the $\pi_{i}$ 's satisfy the property $\left(P_{3}\right)$.

\subsection{Main Results}

We consider $\left\{\pi_{1}, \ldots, \pi_{m}\right\}$ a set of curves and simple closed curves satisfying the properties $\left(P_{1}\right),\left(P_{2}\right)$ and $\left(P_{3}\right)$ defined above. It follows from Subsection 4.2 that such a set of curves can always be constructed. We denote $\pi_{i}=\left(e_{1}^{i}, \ldots, e_{q_{i}}^{i}\right)$ and $e_{s}^{i}=\left(r_{s}^{i}, l_{s}^{i}\right)$ for $i=1, \ldots, m$ and $s=1, \ldots, q_{i}$.

As when we have defined the free group $\mathcal{F}_{m}$, we consider the alphabet

$$
\left\{a_{1}, \ldots, a_{m}, a_{1}^{-1}, \ldots, a_{m}^{-1}\right\}
$$

with $2 m$ letters, and $\mathcal{W}_{m}$ the set of all word on this alphabet.

Let $c=\left(x_{0}, \ldots, x_{p}\right)$ be any $e$-path in $X$. We want to construct a word $w(c) \in \mathcal{W}_{m}$ associated with $c$.

Definition 10. Let $k \in\{0, \ldots, p-1\}, i \in\{1, \ldots, m\}$ and $s \in\left\{1, \ldots, q_{i}\right\}$, be such that $\left\{r_{s}^{i}, l_{s}^{i}\right\}=\left\{x_{k}, x_{k+1}\right\}$. The triple $(k, i, s)$ is called an intersection of $c$ and the $\pi_{j}$ 's. We say that this intersection is positive if $\left(r_{s}^{i}, l_{s}^{i}\right)=\left(x_{k}, x_{k+1}\right)$, and negative if $\left(l_{s}^{i}, r_{s}^{i}\right)=\left(x_{k}, x_{k+1}\right)$.

Notation: Given $(k, i, s)$ an intersection of $c$ and the $\pi_{j}$ 's. We denote by $\mathcal{O}_{c}(k, i, s)$ the number equal to +1 if the intersection $(k, i, s)$ is positive, and to -1 otherwise. 
We define $w(c)$ as the word, containing one symbol $a_{i}^{\mathcal{O}_{c}(k, i, s)}$ for each intersection $(k, i, s)$ of $c$ with the $\pi_{j}$ 's, these symbols appearing in the word $w(c)$ in the order of the increasing $k$ (i.e. the order in which the intersections appear along $c$ ). We denote by $\overline{w(c)}$ the class of $w(c)$ up to elementary simplifications (see the definition of the free group).

Now we can define a group $\Gamma$ given by generators and relations. Let us consider a loop $L_{\pi_{i}}(s)$, with $i \in\{1, \ldots, m\}$ and $s \in\left\{0, q_{i}\right\}$ (which is an extremity of the curve $\left.\pi_{i}\right)$. we assume that the loop $L_{\pi_{i}}(s)$ is contained in $X$. As we observed when defining loops, the loop $L_{\pi_{i}}(s)$ admits a parametrization, which is a closed $e$-path covering $L_{\pi_{i}}(s)$. We denote by $c(i, s)$ such a parametrization. We denote by $R(i, s)$ the word $w(c(i, s))$, and by $\mathcal{R}$ the set of all words $R(i, s)$ for all $i \in\{1, \ldots, m\}$ and $s \in\left\{0, q_{i}\right\}$ such that the loop $L_{\pi_{i}}(s)$ is contained in $X$. Note that, since the parametrization $c(i, s)$ of the loop $L_{\pi_{i}}(s)$ is not exactly unique, but depends on an initial surfel and the orientation of a rotation in $L_{\pi_{i}}(s)$, the word $R(i, s)$ is only defined up to cyclic permutation or inversion in the free group. The normal subgroup of $\mathcal{F}_{m}$ generated by $\mathcal{R}$ does not depend on this choice of the parametrization $c(i, s)$ since two cyclic permutations of the same word correspond to conjugate elements of $\mathcal{F}_{m}$.

We denote by $H$ the normal subgroup of the free group $\mathcal{F}_{m}$ generated by the words of $\mathcal{R}$. As in the definition of the quotient group, we denote by $\equiv_{H}$ the relation of equality modulo elements of $H$ in $\mathcal{F}_{m}$. Finally, we denote $\Gamma=\mathcal{F}_{m} / H$ the quotient group, and we denote by $p_{H}: \mathcal{F}_{m} \longrightarrow \Gamma$ the projection. The group $\Gamma$ is the group with $m$ generators and the relations of $\mathcal{R}$. We want to prove that the $e$-fundamental group of $X$ is isomorphic to the group $\Gamma$. More precisely, we have the two following results :

Theorem 4. The map $p_{H} \circ w: A_{B}^{e}(X) \longrightarrow \Gamma$ is constant on each equivalence class of $A_{B}^{e}(X)$ for the e-homotopy relation in $X$. Therefore, this map $p_{H} \circ w$ induces a map

$$
\left\{\begin{aligned}
\varphi: \Pi_{1}^{e}(X) & \longrightarrow \Gamma \\
{[c] } & \longmapsto p_{H}(\overline{w(c)})
\end{aligned}\right.
$$

Obviously, since concatenation of paths corresponds to concatenation of words, this map $\varphi$ is a group morphism.

Theorem 5. The map $\varphi: \Pi_{1}^{e}(X) \longrightarrow \Gamma$ is a group isomorphism.

The idea is that if we consider $(k, i, s)$ with $s \in\left\{0, q_{i}\right\}$ an intersection of an $e$-path $c$ in $X$ and the $\pi_{j}$ 's, and if $c(i, s)$ denotes a parametrization of the loop $L_{\pi_{i}}(s)$, then the closed $e$-path $c(i, s)$, since contained in a loop, is $e$-homotopic in $X$ with fixed extremities to a path reduced to a single surfel. In other words, the element of the $e$-fundamental group of $X$ represented the closed $e-$ path $c(i, s)$ is the unit element of the $e$-fundamental group. Through the isomorphism $\varphi$ from $\Pi_{1}^{e}(X)$ onto $\Gamma=\mathcal{F}_{m} / H$, this is translated into the fact that the element of $\mathcal{F}_{m}$ represented by the word $R(i, s)=w(c(i, s))$ is sent onto the unit element of $\Gamma$. Therefore, $R(i, s)$ must belong to $H$, but this is precisely the definition of $H$. 


\section{Conclusion}

We can compute a presentation for the fundamental group of any subset of a digital surface. Some principles presented here can be reused to study the complete 3D case. However, the ideas of this paper must be widely modified to be used in the 3D case. Mainly, it seems that, in the $3 \mathrm{D}$ case, the curves $\pi_{i}$ 's which we construct must be surfaces in the $3 \mathrm{D}$ space. Of course, the junctions between surfaces are much more difficult to characterize than intersections of curves on a surface. For this reason, the 3D case seems much more difficult than the case of surfaces.

\section{References}

1. G. Bertrand, Simple points, topological numbers and geodesic neighbourhoods in cubic grids, Pattern Rec. Letters, 15, pp 1003-1011, 1994.

2. [2] T. J. Fan, G. Medioni, and R. Nevata. Recognising 3d Objects using Surface Descriptions. IEEE Transactions on Pattern Analysis and Machine Intelligence, 1111, pp 1140-1157, 1989.

3. G.T. Herman, Discrete Multidimensional Jordan Surfaces. CVGIP : Graphical Models and Image Processing, 54(6) pp 507-515, November 1992.

4. T. Y. Kong, A Digital Fundamental Group Computer and Graphics, 13, pp 159166, 1989.

5. T. Y. Kong, Polyhedral Analogs of Locally Finite Topological Spaces R. M. Shortt editor, General Topology and Applications: Proceedings of the 1988 North-east Conference, Middletown, CT (USA), Lecture Notes in Pure and Applied Mathematics, 123, pp 153-164, 1990.

6. A. Lenoir, Fast Estimation of Mean Curvature on the Surface of a 3D Discrete Object, Proceeding of DGCI'97, Lecture Notes in Computer Science 1347, pp 175-186, 1997.

7. R. Malgouyres, Homotopy in 2-dimensional Digital Images, Proceeding of DGCI'97, Lecture Notes in Computer Science 1347, pp 213-222, 1997.

8. R. Malgouyres, Homotopy in 2-dimensional Digital Images, Theoretical Computer Science, to appear.

9. R. Malgouyres, A. Lenoir, Topology Preservation Within Digital Surfaces, Proceedings of Computer Graphics and Image Processing, Warsaw, May 1998, Machine GRAPHICS \& VISION, vol 7, Nos 1/2, pp 417-425, 1998.

10. J. K. Udupa. Multidimensional digital boundaries. CVGIP: Graphical Models and Image Processing, 56(4), pp 311-323, July 1994. 